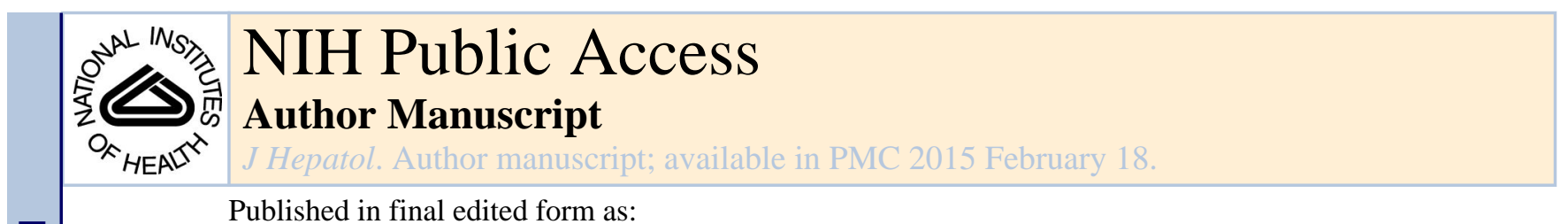

Published in final edited form as:

J Hepatol. 2014 December ; 61(6): 1297-1303. doi:10.1016/j.jhep.2014.07.004.

\title{
Hyper-IL-15 suppresses metastatic and autochthonous liver cancer by promoting tumour-specific CD8+ $T$ cell responses
}

\author{
Liang Cheng ${ }^{1,2, \dagger}$, Xuexiang Du ${ }^{1,2, \dagger}$, Zheng Wang ${ }^{1}$, Jianqi Ju ${ }^{1,2}$, Mingming Jia ${ }^{1}$, Qibin

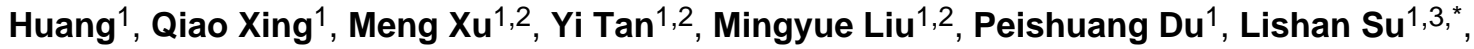 \\ and Shengdian Wang ${ }^{1, *}$ \\ ${ }^{1}$ Key Laboratory of Infection and Immunity, Institute of Biophysics, Chinese Academy of \\ Sciences, Beijing, China \\ ${ }^{2}$ University of Chinese Academy of Sciences, Beijing, China \\ ${ }^{3}$ Lineberger Comprehensive Cancer Center, School of Medicine, The University of North Carolina \\ at Chapel Hill, Chapel Hill, NC, USA
}

\begin{abstract}
Background \& Aims-Liver cancer has a very dismal prognosis due to lack of effective therapy. Here, we studied the therapeutic effects of hyper-interleukin15 (hyper-IL-15), which is composed of IL-15 and the sushi domain of the IL-15 receptor a chain, on metastatic and autochthonous liver cancers.
\end{abstract}

Methods-Liver metastatic tumour models were established by intraportally injecting syngeneic mice with murine CT26 colon carcinoma cells or B16-OVA melanoma cells. Primary hepatocellular carcinoma (HCC) was induced by diethylnitrosamine (DEN). A hydrodynamicsbased gene delivery method was used to achieve sustained hyper-IL-15 expression in the liver.

Results-Liver gene delivery of hyper-IL-15 robustly expanded CD8 ${ }^{+} \mathrm{T}$ and NK cells, leading to a long-term (more than 40 days) accumulation of $\mathrm{CD} 8^{+} \mathrm{T}$ cells in vivo, especially in the liver. Hyper-IL-15 treatment exerted remarkable therapeutic effects on well-established liver metastatic tumours and even on DEN-induced autochthonous HCC, and these effects were abolished by depletion of $\mathrm{CD}^{+} \mathrm{T}$ cells but not NK cells. Hyper-IL-15 triggered IL-12 and interferon- $\gamma$ production and reduced the expression of co-inhibitory molecules on dendritic cells in the liver. Adoptive transfer of T cell receptor (TCR) transgenic OT-1 cells showed that hyper-IL-15 preferentially expanded tumour-specific $\mathrm{CD}^{+} \mathrm{T}$ cells and promoted their interferon- $\gamma$ synthesis and cytotoxicity.

\footnotetext{
(C) 2014 European Association for the Study of the Liver. Published by Elsevier B.V. All rights reserved.

"Corresponding authors. Addresses: Lineberger Comprehensive Cancer Center, CB\#7295, Chapel Hill, NC 27599, USA. Tel: +1 919 966 6654; fax: +1 9199668212 (L Su). Institute of Biophysics, 15 Datun Road, Chaoyang District, Beijing 100101, China. Tel: +86 10 64888493; fax: +86 1064846538 (S. Wang)., 1su@emed.unc.edu (L. Su), sdwang@ moon.ibp.ac.cn (S. Wang).

${ }^{\dagger}$ These authors contributed equally to the work.

Conflict of interest

The authors who have taken part in this study declared that they do not have anything to disclose regarding funding or conflict of interest with respect to this manuscript.

Supplementary data

Supplementary data associated with this article can be found, in the online version, at http://dx.doi.org/10.1016/j.jhep.2014.07.004.
} 
Conclusions-Liver delivery of hyper-IL-15 provides an effective therapy against wellestablished metastatic and autochthonous liver cancers in mouse models by preferentially expanding tumour-specific $\mathrm{CD}^{+} \mathrm{T}$ cells and promoting their anti-tumour effects.

\section{Keywords}

Liver cancer; Immunotherapy; IFN- $\gamma$; Cytotoxicity

\section{Introduction}

Hepatocellular carcinoma (HCC) has a very poor prognosis [1,2]. Traditional therapies for HCC such as surgery are limited by tumour size and intrahepatic metastases [3]. The only clinically approved chemotherapy drug for advanced HCC is sorafenib, which shows only modest efficacy, improving survival of patients by just 3 months [3,4]. Therefore, there is an urgent need to develop more effective therapeutic strategies for liver cancer. Accumulating evidence shows that immunotherapy might become a potential therapeutic option for patients with HCC [5]. Adoptive transfer of autologous T lymphocytes, stimulated by antiCD3 and IL-2, into patients with HCC significantly improved post-surgical recurrence-free survival [6]. Recently, Sangro et al. showed attractive evidence for the application of CTLA-4 targeted immunotherapy in patients with HCC [7].

Administration of cytokines to enhance immune responses has proven to be an effective immunotherapy for some cancers [8]. IL-2 has been used to treat metastatic melanoma patients $[9,10]$. IL-15, a cytokine that shares many features with IL-2, including the use of two receptor subunits (IL-2R $\beta$ and IL-2R $\gamma$ ) and similar intracellular signalling, also shows anti-tumour activity in mice [10]. IL-15 promotes proliferation and effector function of $\mathrm{CD}^{+} \mathrm{T}$ cells, natural killer (NK) cells and NKT cells [10]. More attractively, IL-15 shows no obvious adverse effects in vivo, such as T-cell activation-induced cell death or expansion of T regulatory cells, compared to IL-2 [10]. Trans-presentation of IL-15 by IL-15Ra on activated monocytes and dendritic cells to the IL-2R/IL-15R $\beta$ and $\gamma$ chain on effector T, B, and NK cells is thought to be the primary mechanism for IL-15 action [11,12]. Recent reports have shown that administration of preformed complexes of IL-15 with its soluble receptor IL-15Ra (amino acids 1-78) enhanced the bioavailability of IL-15 by about 50 -fold and showed elevated tumour-eliminating effects in vivo [13-16]. This complex led to an extended half-life of IL-15 in vivo and robust proliferation of antigen-experienced CD44 ${ }^{\text {hi }}$ $\mathrm{CD}^{+} \mathrm{T}$ cells, NK cells and NKT cells [13-16]. Importantly, the soluble fusion protein of IL-15Ra (amino acids 1-78) and IL-15, linked by a flexible pep-tide, exhibited enhanced activity relative to non-covalently associated IL-15 and sIL-15Ra in vitro and in vivo $[17,18]$.

Considerable efforts have been mounted towards testing the anti-tumour activity of the IL-15/IL-15Ra-Fc complex or fusion protein in several cancer models in mice $[13,15,18,19]$. However, its therapeutic benefit for HCC has not been clearly defined. The idea of targeting the IL-15 pathway to treat liver cancer is further supported by the finding that higher IL-15 protein levels in peritumoural liver tissues are significantly associated with better prognosis in patients with resected HCC [20]. Chang et al. recently reported the 
therapeutic use of AAV8-delivered IL-15 superagon-ist (IL-15/IL-15RaS) in a mouse model of metastatic HCC [21]. However, this treatment was only shown to be effective when administered at very early time points ( 3 days after intrasplenic injection of tumour cells), limiting the translational relevance. The effects of a IL-15 superagonist on more established, clinically relevant tumours, such as autochthonous HCC models [22], remains unknown.

In this study, we used the hydrodynamics-based gene delivery method to express a fusion protein of soluble IL-15Ra, fused with IL-15 and human IgG1-Fc (designated as hyperIL-15 hereafter) in vivo to treat liver cancers. We report that ectopic hyper-IL-15 expression had significant therapeutic effects on both well-established metastatic and autochthonous liver cancers in mice, and these effects were primarily mediated by $\mathrm{CD} 8^{+} \mathrm{T}$ cells.

Mechanistically, hyper-IL-15 could preferentially expand tumour-specific $\mathrm{CD} 8^{+} \mathrm{T}$ cells and enhance their cytotoxic activity. Our results have significant implications for the application of hyper-IL-15 to immunotherapeutic intervention of metastatic or autochthonous liver cancers in humans.

\section{Materials and methods}

\section{Experimental animals}

Female C57BL/6 (B6) and BALB/c mice (aged 6-8 weeks) were obtained from Weitong Lihua (Beijing, China). OT-1/Thy1.1 mice were obtained by backcrossing B6 Thy1.1 and OT-1 mice purchased from Jackson Laboratory. All mice were maintained in a specific pathogen-free barrier facility at the Institute of Biophysics. All animal studies were approved by the Institutional Laboratory Animal Care and Use Committee.

\section{Antibodies and reagents}

The fluorescently-labelled anti-mouse NK1.1, CD3, DX5, CD4, CD8, B7-H1, FoxP3, CD25, CD11b, CD11c, CD90.1 (Thy1.1), and IFN- $\gamma$ antibodies, brefeldin A solution and Cytofix/Cytoperm ${ }^{\mathrm{TM}}$ kit were purchased from eBioscience (San Diego, CA). Rabbit antiasialo GM1 (a-GM1) antiserum and control rabbit serum were purchased from Wako Pure Chemical (Tokyo, Japan). CD8 ${ }^{+} \mathrm{T}$ cell depletion antibody (a-CD8, clone TIB210), NK1.1 ${ }^{+}$ cell depletion antibody (a-NK1.1, clone PK136) and rat anti-KLH mAb (rat IgG2a) were purified from ascites of nude mice. $\mathrm{H}-2 \mathrm{~K}^{\mathrm{b}}$ tetramer SIINFEKL-PE was purchased from Beckman Coulter.

\section{Vector construction, recombinant protein preparation}

Construction of the hIgG-Fc (Fc), mouse hyper-IL-15-Fc (hyper-IL-15), IL-15 and IL-15-Fc expression cassettes is shown in Supplementary Fig. 1A. The proteins were prepared by transient transfection of $293 \mathrm{~T}$ cells and purified by protein $\mathrm{G}$ columns.

\section{Hydrodynamic-based gene delivery}

For each mouse, $10 \mu \mathrm{g}$ DNA was diluted in $2.0 \mathrm{ml}$ of PBS ( $0.1 \mathrm{ml} / \mathrm{g}$ body weight) and injected into the tail vein using a 27-gauge needle over 5 to $8 \mathrm{~s}$. In vivo gene expression was confirmed by detecting the protein in the serum by ELISA. 


\section{Metastatic or autochthonous liver cancer models}

Metastatic liver tumours were established by injecting $1 \times 10^{5}$ CT26 or $3 \times 10^{5}$ B16-OVA tumour cells in $150 \mu \mathrm{PBS}$ solution into mice through the portal vein using a $32 \mathrm{G}$ needle. To induce autochthonous liver cancers, 15-day-old male C57BL/6 mice were injected intraperitoneally (i.p.) with $25 \mathrm{mg} / \mathrm{kg}$ DEN (Sigma, St. Louis) dissolved in DMSO. Visible liver nodules were counted and nodule size was measured with calipers by measuring two perpendicular.

\section{Flow cytometry}

Splenocytes and intra-hepatic lymphocytes (IHLs) were prepared as described [23]. Cells were pre-incubated with anti-CD16/32 mAb and then stained with surface or intracellular markers. Flow cytometry was performed on FACSCalibur (BD Bioscience, San Jose, CA) and data were analysed with FlowJo software (TreeStar, Ashland, OR).

\section{In vivo cytotoxicity assay}

The in vivo cytotoxicity assay was performed as described in the Supplementary Materials and methods and as described previously [24].

\section{Histology and immunohistochemistry}

The paraffin embedded tissues were sliced $(5 \mu \mathrm{m})$ and stained with haematoxy-lin-eosin. Frozen sections $(5 \mu \mathrm{m})$ were stained with rat anti-CD8a (eBioscience, San Diego, CA) or rat IgG2a control antibody (eBioscience, San Diego, CA) and then developed with the Polink-1 HRP detection system for rat primary antibodies (ZSGB-BIO, Beijing). The slides were scanned with a Leica SCN 400 (Leica Camera, Allendale, NJ) and images were analysed by using SlidePath Gateway (Leica Microsystems Inc.).

\section{Statistics}

Statistical analysis was performed using the two-tailed, unpaired Student's $t$ test ( ${ }^{*} p<0.05$; $* * p<0.01 ; * * * p<0.001 ; \mathrm{ns}$, non-significant) using GraphPad Prism 5 software (GraphPad Software, La Jolla, CA).

\section{Results}

\section{Hyper-IL-15 robustly expands CD8+ $T$ cells and NK cells in the liver}

To evaluate the therapeutic effect of hyper-IL-15 on liver cancer, we used hydrodynamicsbased gene delivery, which achieves high expression of plasmid DNA by hepatocytes [25]. After hydrodynamic injection of the hyper-IL-15 plasmid, the level of hyper-IL-15 in the plasma reached a peak at day 1 and then decreased over time, reaching low levels after around 2 weeks (Supplementary Fig. 2A). At day 4 after hydrodynamic injection, the total number of intrahepatic lymphocytes (IHLs) increased up to 70-fold in hyper-IL-15 treated mice compared with the Fc-treated control group. The absolute numbers of $\mathrm{CD} 8^{+} \mathrm{T}$ cells and NK cells were 200-fold higher in the hyper-IL-15 group than that in the control group, while $\mathrm{CD} 4^{+} \mathrm{T}$ cells were just slightly increased in hyper-IL-15-treated mice. Correspondingly, the percentages of $\mathrm{CD} 8^{+} \mathrm{T}$ cells and NK cells were significantly 
increased, and the percentages of $\mathrm{CD} 4^{+} \mathrm{T}$ cells were dramatically decreased after hyperIL-15 delivery (Fig. 1A). Similar results were obtained in the spleen (Supplementary Fig. $3 \mathrm{~A})$. In contrast, expression of IL-15 alone only slightly increased the number of $\mathrm{CD} 8^{+} \mathrm{T}$ cells and NK cells in the liver (Supplementary Fig. 3B). Intravenous injection of recombinant hyper-IL-15 protein also significantly increased the number of $\mathrm{CD} 8^{+} \mathrm{T}$ cells and NK cells in liver (Supplementary Fig. 3C). Although hyper-IL-15 treatment induced robust accumulation of $\mathrm{CD} 8^{+} \mathrm{T}$ cells and NK cells in the liver, no obvious liver damage was observed in hyper-IL-15-treated mice compared to control mice as indicated by the alanine aminotransferase (ALT) level in the plasma (Supplementary Fig. 2B).

Next we determined the dynamics of CD8 ${ }^{+} \mathrm{T}$ cells and NK cells expanded by hyper-IL- 15 . As shown in Fig. 1B, the CD8 ${ }^{+} \mathrm{T}$ cell increase, induced by hyper-IL-15 treatment, persisted much longer than that of NK cells in the peripheral blood. At day 40 after the hydrodynamic injection, the percentage and number of $\mathrm{CD}^{+} \mathrm{T}$ cells were still 2-fold higher in the livers of hyper-IL-15-treated mice than in control mice (Fig. 1C). These results demonstrate that hyper-IL-15 markedly expanded $\mathrm{CD}^{+} \mathrm{T}$ cells and NK cells in vivo, leading to a long-term accumulation of $\mathrm{CD} 8^{+} \mathrm{T}$ cells in the liver.

\section{Hyper-IL-15 exerts remarkable anti-tumour effects on liver metastatic tumours}

To study the therapeutic effect of hyper-IL-15 on metastatic liver tumours, we intraportally injected CT26 colon carcinoma cells or B16-OVA melanoma cells into syngeneic mice. Disseminated metastatic tumours in the liver were palpable at day 10 and became extremely serious by day 15 (Supplementary Fig. 4A). We treated the tumour-bearing mice with hyperIL-15 or Fc control plasmids by hydrodynamic injection at day 10 and evaluated the therapeutic effect at day 18 after tumour inoculation. As shown in Fig. 2A and Supplementary Fig. 4B, most mice in the Fc control group showed serious disseminated tumours that had merged together, while only a few scattered tumour nodules were observed in livers of hyper-IL-15-treated mice. The liver weight varied between individual mice in the Fc control group, with livers weighing 1.5 and 2.8 times more on average than those of hyper-IL-15-treated mice in the CT26 and B16-OVA tumour models, respectively. Thus, hyper-IL-15 showed impressive therapeutic effect on well-established metastatic tumours in the liver.

\section{$\mathrm{CD8}^{+} \mathrm{T}$ cells mediate the therapeutic effect of hyper-IL-15}

Similar to the results in tumour-free mice, hyper-IL-15 treatment dramatically increased both $\mathrm{CD}^{+} \mathrm{T}$ cells and NK cells in the livers of tumour bearing mice detected by flow cytometry and immunohistochemical staining (Supplementary Fig. 5). Next, we examined the contribution of each cell population to the suppression of metastatic liver tumours by depleting $\mathrm{CD} 8^{+} \mathrm{T}$ cells or NK cells respectively with antibodies during hyper-IL-15 treatment. The specific depletion effects were verified by flow cytometry analysis of PBMCs (Supplementary Fig. 6). As shown in Fig. 2B, systemic depletion of CD8 ${ }^{+} \mathrm{T}$ cells completely abrogated the therapeutic effects of hyper-IL-15 on metastatic liver CT26 carcinomas, whereas depletion of NK cells had minimal effect. Similar results were obtained in the B16-OVA tumour model (Fig. 2C). These results demonstrate that liver expression of hyper-IL-15 dramatically suppressed growth of liver metastatic tumours, and was mediated 
by $\mathrm{CD} 8^{+} \mathrm{T}$ cells, while expansion of NK cells had negligible effects on the anti-tumour efficacy of hyper-IL-15.

\section{Hyper-IL-15 enhances the cytotoxic function of tumour-specific CD8+ $T$ cells}

Tumour-specific $\mathrm{T}$ cells, which are the major effector cells against tumours, are usually rendered tolerant during tumour development [26]. Here, we examined the effects of hyperIL-15 on tumour-specific $\mathrm{CD}^{+} \mathrm{T}$ cell responses. B6 mice were adoptively transferred with naive thy $1.1^{+}$OT-1 T cells and then intraportally injected with B16-OVA melanoma cells. After 10 days, the mice with palpable metastatic tumours in the liver were treated by hydrodynamic injection of hyper-IL-15 or Fc control vector. Compared with the Fc vector, hyper-IL-15 treatment dramatically increased $\mathrm{CD} 8^{+} \mathrm{T}$ cells by 18.7 and 19.9 fold respectively and, to a greater extent, increased adoptively transferred OT-1 cells by 62.2 and 82.4 fold in liver and spleen, respectively, in B16-OVA tumour-bearing mice (Fig. 3A). Correspondingly, the percentage of OT- 1 cells in $\mathrm{CD}^{+} \mathrm{T}$ cells was 3.2 and 4.4 fold higher in the liver and spleen of hyper-IL-15-treated mice compared to Fc-treated control mice (Fig. 3A). This result suggests that hyper-IL-15 preferentially expanded tumour-specific CD8 ${ }^{+} \mathrm{T}$ cells.

To analyse the cytotoxicity of tumour-specific $\mathrm{CD}^{+} \mathrm{T}$ cells in vivo, the hyper-IL- 15 or Fctreated tumour-bearing mice were intravenously injected with a 1:1 mixture of spleen cells, pulsed with either OVA-257 peptide (SIINFEKL) or control HBV-S208 peptide (ILSPFLPL), which was labelled with carboxyfluorescein succinimidyl ester (CFSE) in high or low concentrations respectively, to serve as target cells. Splenocytes were collected $6 \mathrm{~h}$ thereafter to analyse CFSE-labelled cells for cytotoxicity determination. As shown in Fig. 3B, the effective elimination of OVA peptide-loaded cells was observed in hyper-IL-15 treated mice but not in the control group. The mean specific lysis in the hyper-IL-15 treated group was $80 \%$, significantly higher than the observed $4 \%$ in the Fc-treated group. Thus, hyper-IL-15 markedly promoted the cytotoxic activity of tumour-specific $\mathrm{CD} 8^{+} \mathrm{T}$ cells in vivo.

To further analyse the effect of hyper-IL-15 on the function of tumour-specific CD8 ${ }^{+} \mathrm{T}$ cells, we isolated OT-1 splenocytes from naive or tumour-bearing mice 4 days after hyperIL-15 treatment and treated them with OVA-257 peptide ex vivo. The IFN- $\gamma$ producing OT-1 $\mathrm{T}$ cells were detected by flow cytometry. As shown in Fig. 3C, thy $1.1^{+} \mathrm{OT}-1 \mathrm{~T}$ cells from tumour naive mice could be stimulated by OVA peptide to produce IFN- $\gamma$, and hyper-IL- 15 treatment markedly increased the IFN- $\gamma$ producing OT-1 T cells in the spleen of naive mice. However, OT-1 cells from the Fc-treated tumour-bearing mice did not react to OVA peptide stimulation, and the percentage of IFN- $\gamma^{+}$cells in OT-1 splenocytes was only $5 \%$ in comparison with $25 \%$ in naive mice. This result indicated that tumour-specific CD8 ${ }^{+} \mathrm{T}$ cells were functionally deficient or tolerant in mice with metastatic liver tumours. Impressively, OT-1 T cells from hyper-IL-15-treated tumour-bearing mice responded well to OVA peptide stimulation, with $45 \%$ of spleen OT- 1 cells producing IFN- $\gamma$, which was similar to the $47 \%$ of IFN- $\gamma^{+}$OT-1 T cells from hyper-IL-15-treated naive mice (Fig. 3C). Thus, tumourspecific OT-1 cells in tumour-bearing mice were fully activated after hyper-IL-15 treatment. 
These results demonstrate that hyper-IL-15 treatment not only expanded the number of tumour-specific $\mathrm{CD} 8^{+} \mathrm{T}$ cells, but also greatly enhanced their anti-tumour activity in mice with liver metastatic tumours.

\section{Hyper-IL-15 treatment triggers pro-inflammatory cytokine production and significantly increases the ratio of $\mathrm{CD}^{+} \mathrm{T}$ cells vs. Treg cells in the liver}

We also tested whether hyper-IL-15 treatment triggered other pro-inflammatory cytokines important for tumour elimination. As shown in Fig. 3D, hyper-IL-15 induced robust IL-12 and IFN- $\gamma$, but not IL-10, in the plasma of tumour bearing mice 4 days after treatment. In parallel with tumour growth, expression of the co-inhibitory molecule B7-H1 on dendritic cells $\left(\mathrm{CD} 45^{+} \mathrm{CD} 11 \mathrm{~b}^{+} \mathrm{CD} 11 \mathrm{c}^{+}\right)$was upregulated, and regulatory $\mathrm{CD} 4^{+} \mathrm{T}$ cells (Treg cells) accumulated in the liver (Supplementary Fig. 4C). Hyper-IL-15 treatment could downregulate the expression of B7-H1 on dendritic cells (Fig 3E). Although hyper-IL-15 treatment expanded intrahepatic $\mathrm{T}$ cells in general, including $\mathrm{CD}^{+} \mathrm{T}$ cells, the percentage of Treg cells in the $\mathrm{CD} 4^{+} \mathrm{T}$ cell population and the overall number of Treg cells was not significantly increased in liver (Fig 3E). Correspondingly, the ratio of $\mathrm{CD}^{+} \mathrm{T}$ cells, which underwent strong expansion, $v s$. regulatory $\mathrm{T}$ cells, which did not, was dramatically increased by hyper-IL-15 treatment (Fig 3E). These data suggested that hyper-IL-15 treatment activated anti-tumour immune pathways without inducing immune suppressive pathways in liver.

\section{Hyper-IL-15 shows significant therapeutic effect on autochthonous hepatocellular carcinoma}

We further studied the potential of hyper-IL-15 for clinical application by using the diethylnitrosamine (DEN)-induced autochthonous hepatocarcinogenesis model in which malignant cells mostly arise from normal indigenous hepatocytes and grow slowly in immunocompetent mice. Male mice were intraperitoneally injected with DEN 15 days after birth and HCC developed within 8 months. Starting at 8.5 months, the mice were given two rounds of hydrodynamic injections of hyper-IL-15 or Fc plasmids with an interval of two weeks. Two months after the second treatment, all mice underwent hepatectomy. Mice treated with Fc exhibited massive multi-nodular HCCs, while mice treated with hyper-IL-15 had only a few small, sporadic tumour nodules (Fig. 4A). The mean largest tumour volume and the total volume of all tumours per mouse were respectively about 80 -fold and 100 -fold smaller in hyper-IL-15-treated mice than that in Fc-treated mice (Fig. 4B). Depletion of $\mathrm{CD} 8^{+} \mathrm{T}$ cells by antibody administration during treatment partially diminished the therapeutic effects of hyper-IL-15 (Fig. 4A-C). Tumour nodules in the liver were confirmed by histochemical staining (Fig. 4C).

Taken together, our studies demonstrate that liver delivery of hyper-IL-15 has significant therapeutic effects on both metastatic and autochthonous liver cancers.

\section{Discussion}

We demonstrated here that liver gene delivery of hyper-IL-15 not only dramatically inhibited the growth of well-established liver metastatic tumours, but also exerted a 
remarkable therapeutic effect on primary hepatocellular carcinoma. The anti-tumour effect of hyper-IL-15 was mediated mainly by $\mathrm{CD}^{+} \mathrm{T}$ cells, but not by NK cells. We found that hyper-IL-15 could enormously expand $\mathrm{CD} 8^{+} \mathrm{T}$ cells in vivo, cause long-term accumulation of $\mathrm{CD} 8^{+} \mathrm{T}$ cells in the liver, and markedly promote functions of tumour-specific $\mathrm{CD} 8^{+} \mathrm{T}$ cells.

Although hyper-IL-15 tremendously expanded both CD8 ${ }^{+} \mathrm{T}$ cells and NK cells in vivo, the results of lymphocyte deletion showed that the anti-tumour effects of hyper-IL-15 were dependent on $\mathrm{CD}^{+}{ }^{+} \mathrm{T}$ cells rather than NK cells. This was consistent with the more durable accumulation of $\mathrm{CD} 8^{+} \mathrm{T}$ cells relative to $\mathrm{NK}$ cells in the liver. Chang et al. recently reported that the therapeutic effect of AAV-delivered hyper-IL-15 on metastatic murine liver cancer was mediated by NK cells rather than T cells [21]. In their study, mice were treated with AAV8/IL-15-IL-15RaS three days after tumour inoculation. At this time point, the tumours might not be well-established in the liver. In addition, the AAV itself may have triggered specific pathways to induce innate and adaptive immune responses against both viruses and tumours [27], complicating the interpretation of these experiments with respect to the effects of IL-15/IL-15Ra. Here, we show the impressive therapeutic effect of hyper-IL-15 to wellestablished tumours in the liver 10 days after transplantation. Most importantly, we demonstrate that hyper-IL-15 dramatically inhibited the growth of well-developed DENinduced primary HCC. To our knowledge, this is the first immunotherapy, which shows significant therapeutic effects on DEN-induced autochthonous HCC.

Liver is recognized as a tolerogenic organ [28]. T cells that recognize antigens in the liver are usually exposed to inhibitory ligands, including B7-H1, and to immunosuppressive cytokines, including TGF- $\beta 1$ and IL-10, which are expressed by liver antigen presenting cells such as dendritic cells, Kupffer cells, liver sinusoidal endothelial cells (LSECs) and even hepatocytes $[29,30]$. Thus, the indigenous tolerogenic hepatic environment potentially hinders HCC-directed immunotherapy. It was reported that the IL-15/IL-15Ra complex or fusion protein promoted the destruction of established pancreatic tumours by reviving tumour-resident $\mathrm{CD} 8^{+} \mathrm{T}$ cells and eliminated well-established myeloma by stimulating $\mathrm{CD}^{+} \mathrm{T}$ cells to produce IFN $-\gamma[15,19]$. In our liver metastatic B16-OVA melanoma model, tumour-specific OT-1 cells failed to respond to OVA peptide stimulation ex vivo, suggesting a tolerant state. Hyper-IL-15 therapy preferentially expanded tumour-specific OT-1 cells, and rescued their cytokine production ability and cytotoxic activity. We also found that hyper-IL-15 treatment triggered the production of IFN- $\gamma$ and IL-12, but not IL-10, in tumour bearing mice. The expression of the co-inhibitory molecule B7-H1 on dendritic cells in the liver was also lower in the hyper-IL-15 treated mice compared to controls. These results demonstrated that, in the tolerogenic liver environment, hyper-IL-15 therapy could rescue tolerant tumour-specific $\mathrm{CD} 8^{+} \mathrm{T}$ cells to eliminate tumours.

In conclusion, our study showed that liver delivery of hyper-IL-15 had a remarkable therapeutic effect on liver metastatic tumours and even on primary HCCs by enhancing tumour-specific $\mathrm{CD} 8^{+} \mathrm{T}$ cell responses. Our encouraging results warrant future exploration of the use of hyper-IL-15 for treating metastatic and spontaneous liver cancers in humans. 


\section{Supplementary Material}

Refer to Web version on PubMed Central for supplementary material.

\section{Acknowledgments}

We thank Dr. Christopher Murphy (The University of North Carolina at Chapel Hill, Chapel Hill, NC) for critical reading and editing of the manuscript.

Financial support

This work was supported by grants from the National Key Technologies Research and Development Program of China (2012ZX10002007-001-007), the National Key Basic Research Program of China (No. 2012CB917101), and the National Natural Science Foundation of China (Grant No. 91029719 and 81000920).

\section{Abbreviations}

$\begin{array}{ll}\text { IL-15 } & \text { interleukin-15 } \\ \text { IFN- } \gamma & \text { interferon- } \gamma \\ \text { HCC } & \text { hepatocellular carcinoma } \\ \text { DEN } & \text { diethylnitrosamine } \\ \text { NK cells } & \text { natural killer cells } \\ \text { NKT cells } & \text { natural killer T cells } \\ \text { CFSE } & \text { carboxyfluorescein succinimidyl ester } \\ \text { IHLs } & \text { intrahepatic lymphocytes } \\ \text { PBMC } & \text { peripheral blood mononuclear cell }\end{array}$

\section{References}

1. El-Serag HB, Marrero JA, Rudolph L, Reddy KR. Diagnosis and treatment of hepatocellular carcinoma. Gastroenterology. 2008; 134:1752-1763. [PubMed: 18471552]

2. EASL-EORTC clinical practice guidelines. Management of hepatocellular carcinoma. J Hepatol. 2012; 56:908-943. [PubMed: 22424438]

3. de Lope CR, Tremosini S, Forner A, Reig M, Bruix J. Management of HCC. J Hepatol. 2012; 56:S75-S87. [PubMed: 22300468]

4. Llovet JM, Ricci S, Mazzaferro V, Hilgard P, Gane E, Blanc JF, et al. Sorafenib in advanced hepatocellular carcinoma. N Engl J Med. 2008; 359:378-390. [PubMed: 18650514]

5. Breous E, Thimme R. Potential of immunotherapy for hepatocellular carcinoma. J Hepatol. 2011; 54:830-834. [PubMed: 21145836]

6. Takayama T, Sekine T, Makuuchi M, Yamasaki S, Kosuge T, Yamamoto J, et al. Adoptive immunotherapy to lower postsurgical recurrence rates of hepato-cellular carcinoma: a randomised trial. Lancet. 2000; 356:802-807. [PubMed: 11022927]

7. Sangro B, Gomez-Martin C, de la Mata M, Inarrairaegui M, Garralda E, Barrera P, et al. A clinical trial of CTLA-4 blockade with tremelimumab in patients with hepatocellular carcinoma and chronic hepatitis C. J Hepatol. 2013; 59:81-88. [PubMed: 23466307]

8. Smyth MJ, Cretney E, Kershaw MH, Hayakawa Y. Cytokines in cancer immunity and immunotherapy. Immunol Rev. 2004; 202:275-293. [PubMed: 15546400] 
9. Rosenberg SA, Yang JC, Topalian SL, Schwartzentruber DJ, Weber JS, Parkinson DR, et al. Treatment of 283 consecutive patients with metastatic melanoma or renal cell cancer using highdose bolus interleukin 2. JAMA. 1994; 271:907-913. [PubMed: 8120958]

10. Waldmann TA. The biology of interleukin-2 and interleukin-15: implications for cancer therapy and vaccine design. Nat Rev Immunol. 2006; 6:595-601. [PubMed: 16868550]

11. Dubois S, Mariner J, Waldmann TA, Tagaya Y. IL-15Ralpha recycles and presents IL-15 In trans to neighboring cells. Immunity. 2002; 17:537-547. [PubMed: 12433361]

12. Burkett PR, Koka R, Chien M, Chai S, Boone DL, Ma A. Coordinate expression and trans presentation of interleukin (IL)-15Ralpha and IL-15 supports natural killer cell and memory CD8+ T cell homeostasis. J Exp Med. 2004; 200:825-834. [PubMed: 15452177]

13. Stoklasek TA, Schluns KS, Lefrancois L. Combined IL-15/IL-15Ralpha immunotherapy maximizes IL-15 activity in vivo. J Immunol. 2006; 177:6072-6080. [PubMed: 17056533]

14. Rubinstein MP, Kovar M, Purton JF, Cho JH, Boyman O, Surh CD, et al. Converting IL-15 to a superagonist by binding to soluble IL-15R \{alpha\}. Proc Natl Acad Sci U S A. 2006; 103:91669171. [PubMed: 16757567]

15. Epardaud M, Elpek KG, Rubinstein MP, Yonekura AR, Bellemare-Pelletier A, Bronson R, et al. Interleukin-15/interleukin-15R alpha complexes promote destruction of established tumours by reviving tumour-resident CD8+ T cells. Cancer Res. 2008; 68:2972-2983. [PubMed: 18413767]

16. Dubois S, Patel HJ, Zhang M, Waldmann TA, Muller JR. Preassociation of IL-15 with IL-15R alpha-IgG1-Fc enhances its activity on proliferation of NK and CD8+/CD44high T cells and its antitumour action. J Immunol. 2008; 180:2099-2106. [PubMed: 18250415]

17. Mortier E, Quemener A, Vusio P, Lorenzen I, Boublik Y, Grotzinger J, et al. Soluble interleukin-15 receptor alpha (IL-15R alpha)-sushi as a selective and potent agonist of IL-15 action through IL-15R beta/gamma. Hyperagonist IL-15 $\times$ IL-15R alpha fusion proteins. J Biol Chem. 2006; 281:1612-1619. [PubMed: 16284400]

18. Bessard A, Sole V, Bouchaud G, Quemener A, Jacques Y. High antitumour activity of RLI, an interleukin-15 (IL-15)-IL-1 5 receptor alpha fusion protein, in metastatic melanoma and colorectal cancer. Mol Cancer Ther. 2009; 8:2736-2745. [PubMed: 19723883]

19. Xu W, Jones M, Liu B, Zhu X, Johnson CB, Edwards AC, et al. Efficacy and mechanism-of-action of a novel superagonist interleukin-15: interleukin-15 receptor alphaSu/Fc fusion complex in syngeneic murine models of multiple myeloma. Cancer Res. 2013; 73:3075-3086. [PubMed: 23644531]

20. Zhou H, Huang H, Shi J, Zhao Y, Dong Q, Jia H, et al. Prognostic value of interleukin 2 and interleukin 15 in peritumoural hepatic tissues for patients with hepatitis B-related hepatocellular carcinoma after curative resection. Gut. 2010; 59:1699-1708. [PubMed: 20940284]

21. Chang CM, Lo CH, Shih YM, Chen Y, Wu PY, Tsuneyama K, et al. Treatment of hepatocellular carcinoma with adeno-associated virus encoding interleukin-15 superagonist. Hum Gene Ther. 2010; 21:611-621. [PubMed: 20064014]

22. Ostrand-Rosenberg S. Animal models of tumour immunity, immunotherapy and cancer vaccines. Curr Opin Immunol. 2004; 16:143-150. [PubMed: 15023405]

23. Chen Y, Wei H, Sun R, Dong Z, Zhang J, Tian Z. Increased susceptibility to liver injury in hepatitis B virus transgenic mice involves NKG2D-ligand interaction and natural killer cells. Hepatology. 2007; 46:706-715. [PubMed: 17626270]

24. Narazaki H, Zhu Y, Luo L, Zhu G, Chen L. CD137 agonist antibody prevents cancer recurrence: contribution of CD137 on both hematopoietic and nonhematopoietic cells. Blood. 2010; 115:1941-1948. [PubMed: 20068221]

25. Suda T, Liu D. Hydrodynamic gene delivery: its principles and applications. Mol Ther. 2007; 15:2063-2069. [PubMed: 17912237]

26. Rabinovich GA, Gabrilovich D, Sotomayor EM. Immunosuppressive strategies that are mediated by tumour cells. Annu Rev Immunol. 2007; 25:267-296. [PubMed: 17134371]

27. Hareendran S, Balakrishnan B, Sen D, Kumar S, Srivastava A, Jayandharan GR. Adeno-associated virus (AAV) vectors in gene therapy: immune challenges and strategies to circumvent them. Rev Med Virol. 2013; 23:399-413. [PubMed: 24023004] 
28. Crispe IN. The liver as a lymphoid organ. Annu Rev Immunol. 2009; 27:147-163. [PubMed: 19302037]

29. Thomson AW, Knolle PA. Antigen-presenting cell function in the tolerogenic liver environment. Nat Rev Immunol. 2010; 10:753-766. [PubMed: 20972472]

30. Knolle PA, Limmer A. Neighborhood politics: the immunoregulatory function of organ-resident liver endothelial cells. Trends Immunol. 2001; 22:432-437. [PubMed: 11473832] 

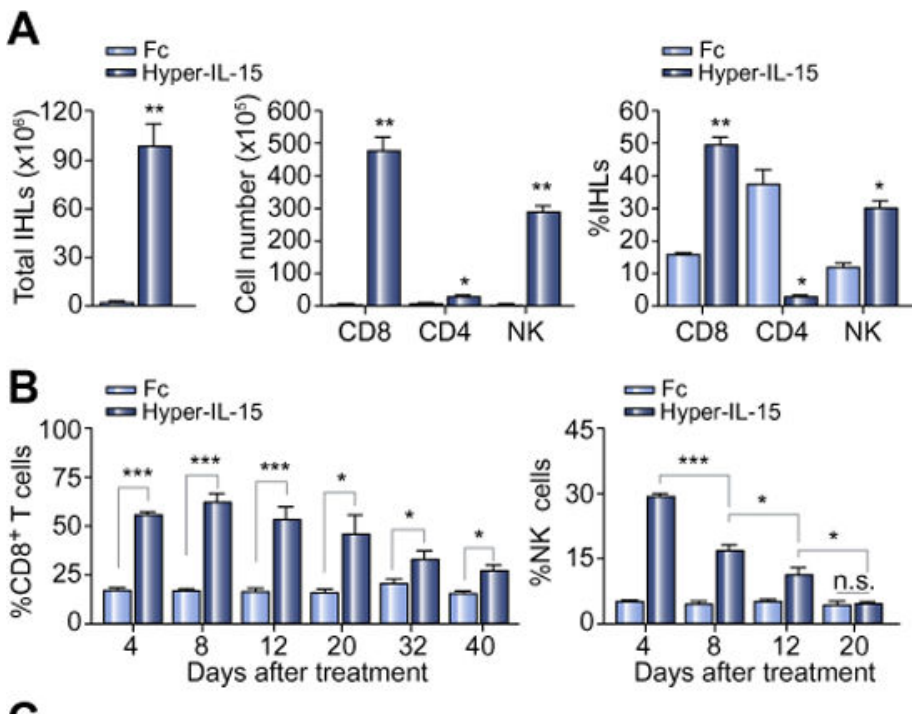

C
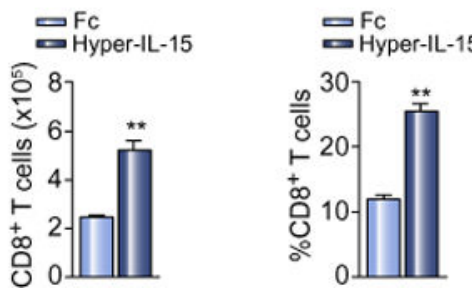

Fig. 1. Liver gene delivery of hyper-IL-15 expands both $\mathrm{CD8}^{+} \mathrm{T}$ cells and NK cells in vivo C57BL/6 mice were hydrodynamically injected with $10 \mu \mathrm{g}$ of hyper-IL-15 or Fc plasmid at day 0. (A) Intrahepatic lymphocytes (IHLs) were isolated for flow cytometry analysis at day 4. Numbers of total IHLs (left), cell numbers (middle) and percentages (right) of $\mathrm{CD} 8^{+} \mathrm{T}$ cells $\left(\mathrm{CD}^{+}{ }^{+} \mathrm{CD}^{+}\right), \mathrm{CD}^{+}{ }^{+} \mathrm{T}$ cells $\left(\mathrm{CD}^{+}{ }^{+} \mathrm{CD} 4^{+}\right)$, NK cells $\left(\mathrm{CD}^{-} \mathrm{NK} 1.1^{+}\right)$in IHLs are shown. Three independent experiments with similar results were performed. (B) Percentages of $\mathrm{CD}^{+} \mathrm{T}$ cells (left) and NK cells (right) in PBMCs at indicated time points. (C) Cell numbers (left) and percentage (right) of $\mathrm{CD} 8^{+} \mathrm{T}$ cells in IHLs in each group at day 40. Graphs represent the mean \pm SEM of 3-5 mice each group in A, B and C. 

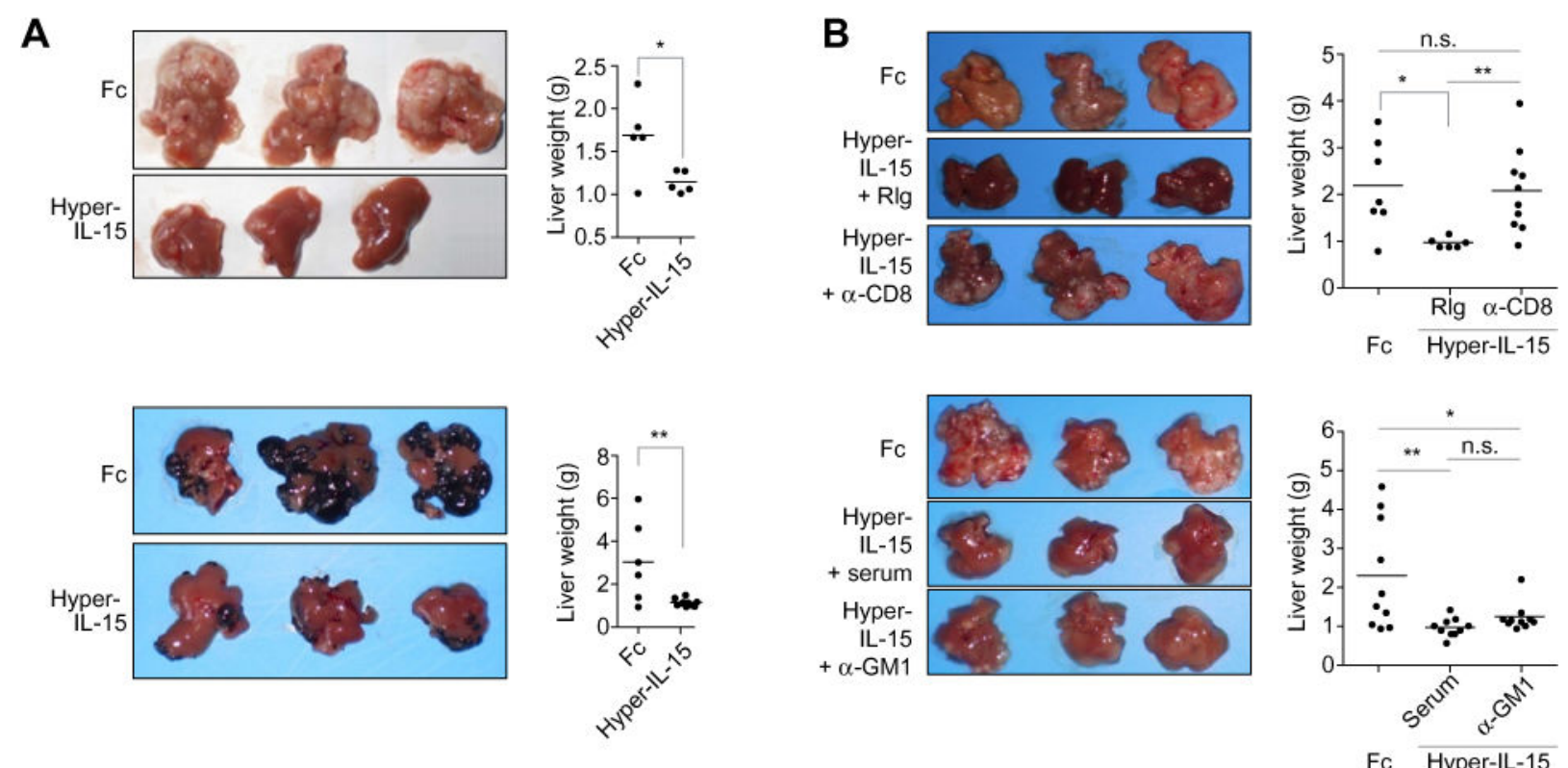

C
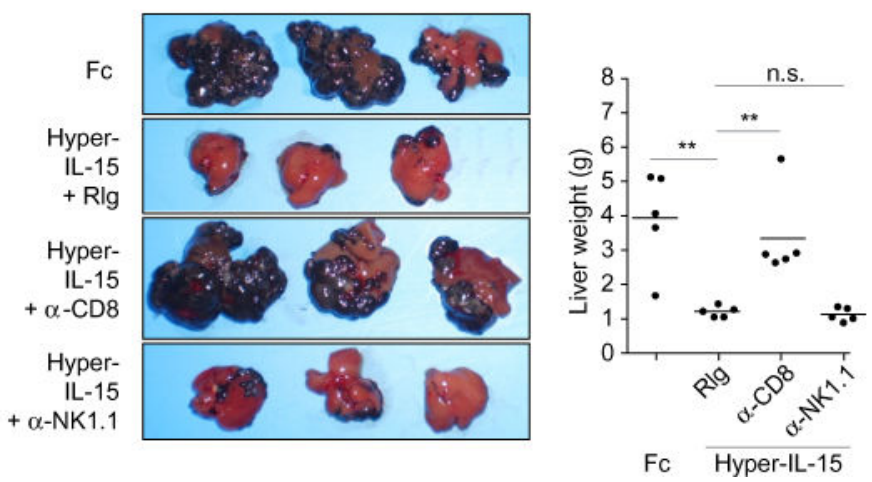

Fig. 2. Therapeutic effect of hyper-IL-15 on liver metastatic tumours

$\mathrm{BALB} / \mathrm{c}$ or C57BL/6 mice were intraportally injected with CT26 tumour cells or B16-OVA melanoma cells and then hydrodynamically injected with hyper-IL-15 or Fc plasmids at day 10. (A) Representative photographs and liver weight with disseminated metastatic CT26 tumours (upper panels) and B16-OVA tumours (bottom panels) at day 18 are shown. Three independent experiments with similar results were performed. (B-C) Some tumour-bearing mice were injected i.p with $200 \mu \mathrm{g} \mathrm{CD}{ }^{+} \mathrm{T}$ cells (a-CD8) or NK cells (a-GM1or a-NK1.1) depletion antibodies 1 day before and 4 days after hydrodynamical injection of the hyperIL-15 plasmid. Representative photographs and liver weight with disseminated metastatic CT26 tumours (B) and B16-OVA tumours (C) at day 18 are shown. Two independent experiments with similar results were performed. Each dot represents one individual mouse and graphs represent the mean of 5-10 mice each group. 
A $=$ B
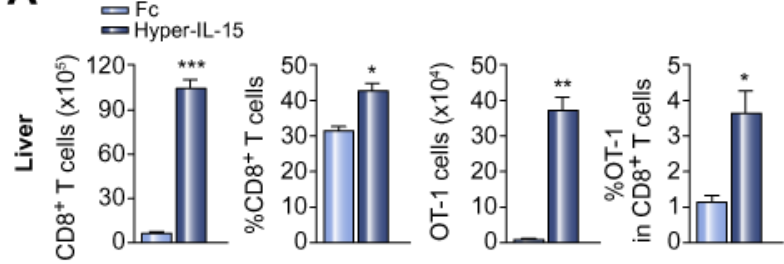

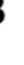
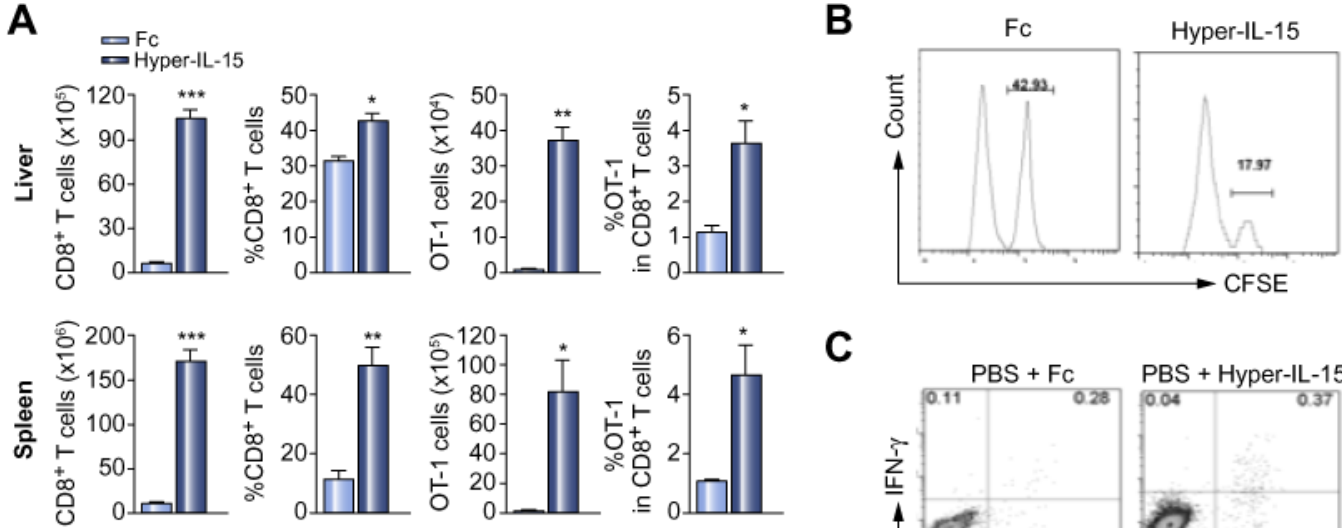

D
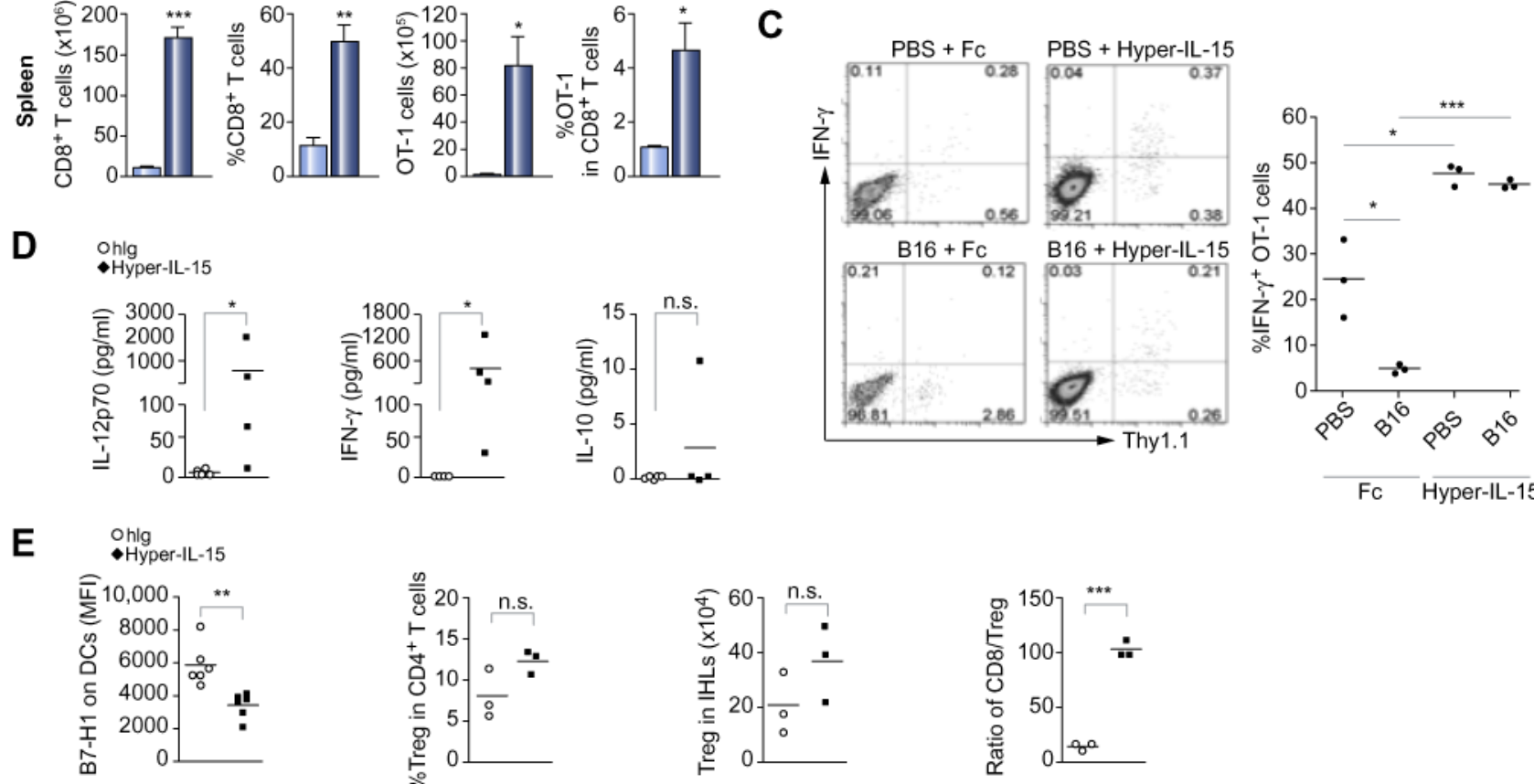

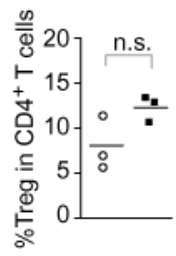

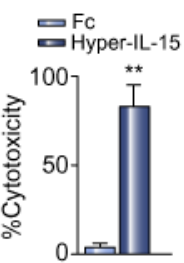

Fc Hyper-IL-15

Fig. 3. Hyper-IL-15 preferentially expands tumour-specific $\mathrm{CD8}^{+} \mathrm{T}$ cells and enhances their cytotoxic function

Thy $1.2^{+}$C57BL/6 mice were adoptively transferred with purified thy $1.1^{+}$OT-1 $\mathrm{T}$ cells one day before they were intraportally injected with B16-OVA cells, followed by hydrodynamic injection with hyper-IL-15 or Fc plasmids 10 days after tumour inoculation. (A) The absolute number and percentage of total $\mathrm{CD} 8^{+} \mathrm{T}$ cells, number of thy $1.1^{+} \mathrm{OT}-1 \mathrm{~T}$ cells and percentage of OT- 1 cells in total $\mathrm{CD}^{+} \mathrm{T}$ cells in the spleen and IHLs are shown. (B) Mice were i.v. injected with a 1:1 mixture of CFSE-labelled spleen cells, pulsed with OVA-257 peptide (right peak) or control HBV-S208 peptide (left peak) 4 days after hyper-IL-15 treatment. After $6 \mathrm{~h}$, splenocytes were isolated and CFSE profiles were analysed. The numbers above the peaks indicate percentages of OVA-257 peptide-pulsed cells among the total CFSE-labelled target cells. Specific lysis of peptide pulsed target cells was calculated. Graphs represent the mean \pm SEM of 3-4 mice each group in A and B. (C) Four days after hyper-IL-15 or Fc treatment, splenocytes were harvested and stimulated ex vivo with 1 $\mathrm{ng} / \mathrm{ml}$ OVA-257 peptide for 2 days and IFN- $\gamma^{+}$-producing cells were detected by intracellular staining. Representative plots of IFN $-\gamma^{+}$OT-1 cell cells are shown (left). Percentages of IFN- $\gamma^{+}$OT- 1 cell were statistically analysed (right). Each dot represents one individual mouse and graphs represent the mean of 3 mice each group. (D-E) C57BL/6 mice were intraportally injected with B16-OVA melanoma cells and then hydrodynamically injected with hyper-IL-15 or Fc plasmids at day 10. At day 14, cytokines IL-12, IFN- $\gamma$ and 
IL-10 in the serum were detected by Luminex assay (D). B7-H1 expression on dendritic cells, percentage and number of Treg cells and $\mathrm{CD} 8^{+} \mathrm{T}$ cells $v$. Treg cells in the liver at day 14 are shown (E). Two independent experiments with similar results were performed. 


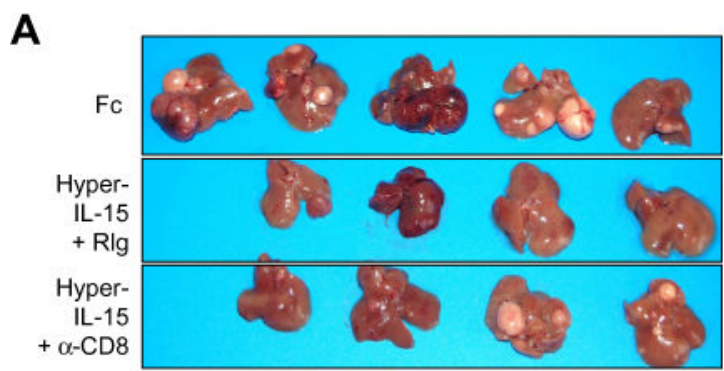

B
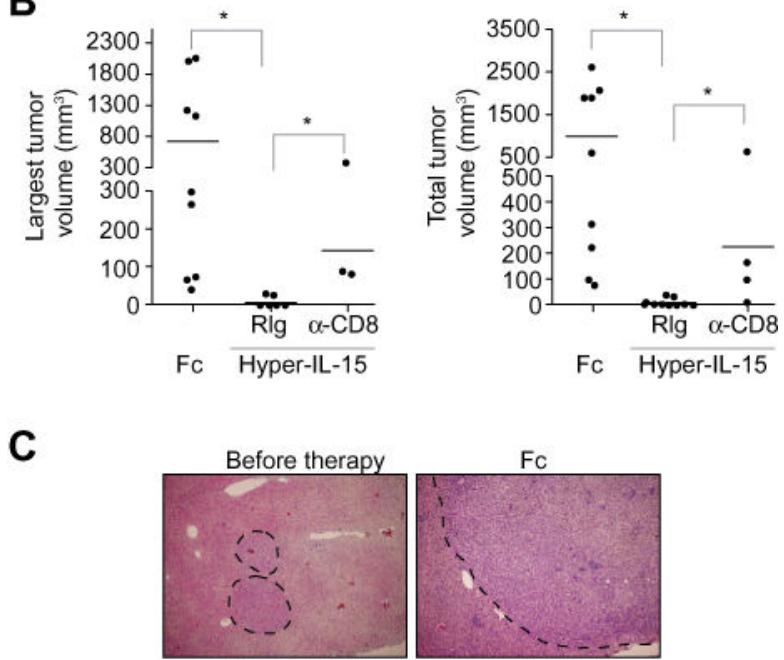

Hyper-IL-15+

Rlg
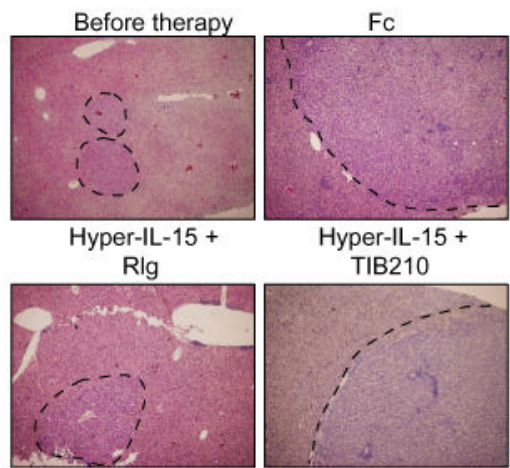

Hyper-IL-15

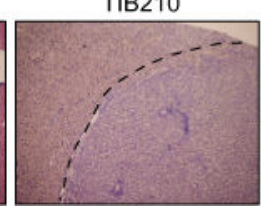

Fig. 4. Therapeutic effect of hyper-IL-15 on DEN-induced autochthonous hepatocellular carcinoma

Male C57BL/6 mice were i.p. injected with DEN 15 days after birth, and hydrodynamically injected with hyper-IL-15 or control Fc plasmids at 8.5 and 9 months. Some hyper-IL-15treated mice were i.p. injected with $200 \mu \mathrm{g}$ of anti-CD8 antibody 1 day before and 4 days after each hydrody-namic injection. At 11 moths, all mice underwent hepatectomy. (A) Representative photograph of livers with HCCs. (B) Size of the largest tumour (left) and total tumour volume (right) in each liver were measured and statistically analysed. Each dot represents one individual mouse and graphs represent the mean of 4-10 mice each group. (C) Representative H\&E staining of liver tissue sections before or after treatment. Black dash lines indicate border of the tumour nodules. 